\title{
Augmented Activity of the Pelvic Nerve Afferent Mediated by TRP Channels in Dextran Sulfate Sodium (DSS)-Induced Colitis of Rats
}

\author{
Yukitoshi MAKIMURA ${ }^{1)}$, Koichi ITO ${ }^{1)}$, Masayoshi KUWAHARA ${ }^{1)}$ and Hirokazu TSUBONE ${ }^{1,2) *}$ \\ 1) Department of Comparative Pathophysiology, Division of Veterinary Medical Sciences, Graduate School of Agricultural and Life \\ Sciences, The University of Tokyo, 1-1-1 Yayoi, Bunkyo-ku, Tokyo 113-8657, Japan \\ ${ }^{2)}$ Research Center for Food Safety, Graduate School of Agricultural and Life Sciences, The University of Tokyo, 1-1-1 Yayoi, Bunkyo- \\ ku, Tokyo 113-8657, Japan
}

(Received 7 December 2011/Accepted 1 April 2012/Published online in J-STAGE 13 April 2012)

ABSTRACT. Enteritis has been recognized as a major symptom in domestic animals and human patients suffering from feed and food poisonings. The aim of the present study was to clarify the excitatory mechanism of the pelvic nerve afferent which may influence the occurrence of enteritis in response to nociceptive chemical stimuli of the colon in normal and abnormal rats with colitis induced by dextran sulfate sodium (DSS). The pelvic nerve afferent activity was markedly increased by colonic instillation of solution $(0.5 \mathrm{~m} l)$ of acetic acid $(5-25 \%)$ and capsaicin $(100 \mu \mathrm{g} / \mathrm{m} l)$. The nerve activity was augmented by colonic instillation of capsaicin to a greater extent in rats with DSS-induced colitis than in normal control rats. This augmented activity by capsaicin was more prominent at one day (DSS-1) than at 8 day (DSS-8) after the administration of DSS. The increased nerve activity caused by capsaicin in DSS-1 and DSS- 8 was significantly inhibited by pretreatment with ruthenium red, which is a nonselective inhibitor of TRP channels of unmyelinated C-fibers (nociceptors). In conclusion, it was elucidated that the nociceptive function of the pelvic nerve was largely elevated at one day after DSS-induced colitis and such increased function was mostly mediated by TRP channels.

KEY WORDS: C-fiber, colitis, nociception, pelvic nerve.

doi: 10.1292/jvms.11-0547; J. Vet. Med. Sci. 74(8): 1007-1013, 2012

Acute enteritis due to food/feed poisonings and chronic enteritis represented by inflammatory bowel disease have been widely recognized as serious health hazards in human patients and domestic animals [2, 8, 20]. Many studies have been conducted on pathophysiological mechanisms of these intestinal diseases, focusing on immunopathologic and microbiological mechanisms, and also on the neural network system in the intestine, using model animals [4, 13, 24]. In these inflammatory disorders, the hyperesthesia to various chemical and mechanical stimuli in the intestine has been known as a major part of symptoms that might be associated with bowel complaint, abnormal intestinal motility and other general symptoms.

Since the neural mechanism of perception in response to intestinal stimuli should play an important role in the physiological regulation of intestinal environments, the inadequate activity of the sensory nerve innervating the intestine is supposed to induce or accelerate inflammatory symptom via mechanisms such as neurogenic inflammation and/or cross-talk actions of neural and immune systems.

It has been known that the sensory innervation of the colon, which is a prevalent region of inflammatory bowel disease, is supplied by the pelvic nerve. However, the de-

\footnotetext{
*Correspondence to: Tsubone, H., Research Center for Food Safety, Graduate School of Agricultural and Life Sciences, The University of Tokyo, 1-1-1 Yayoi, Bunkyo-ku, Tokyo 113-8657, Japan.

e-mail: aahiro@mail.ecc.u-tokyo.ac.jp

(C)2012 The Japanese Society of Veterinary Science
}

tailed electrophysiological properties about the nociceptive perception of this sensory nerve have not been fully explored. The excitability of such nociceptors should contribute to neurogenic inflammation that accelerates or augments pathophysiological conditions of the gastrointestinal tract. It has been considered that unmyelinated $\mathrm{C}$-fibers constitute such nociceptive neurons involving TRPV1-mechanisms being activated by acidic conditions, heat stress greater than $43^{\circ} \mathrm{C}$ and a selective agonist, capsaicin $[1,3,4,16,21]$. It has also been reported that the pelvic nerve of the rat involves C-type fibers at a proportion of $60 \%$ and A $\delta$ fibers at a proportion of $40 \%$, identified by measurement of conduction velocity [4]. These afferent fibers are conceived to play roles in nociceptive chemical and mechanical sensation in the colon. Therefore, it should be important to measure the activity of pelvic nerve afferents and to know the role of nociceptors in inflammation in the lower intestinal tract from clinical aspects regarding bowel diseases and hyperalgesia.

The aim of the present study was to clarify the afferent activity in response to chemical nociceptive stimulation, i.e., with acetic acid and capsaicin, of the colon in normal and abnormal rats with inflammatory bowel caused by treatment with dextran sulfate sodium (DSS) which is a representative inducer of acute colitis with ulcer [12-15].

\section{MATERIALS AND METHODS}

Animals, anesthesia and surgical procedure: Experiments were performed on Wistar Kyoto rats (male, 8-10 weeks, 200-300 g body weight). The rats were housed in 
a temperature-controlled $\left(22-24^{\circ} \mathrm{C}\right)$ room and given water and commercial chow. They were anesthetized with an intraperitoneal injection of urethane $(1.0 \mathrm{~g} / \mathrm{kg})$ and placed supine on an operating table. The femoral vein was exposed and incised in order to place a cannula for drug administration. A polyethylene catheter filled with saline was also inserted into the colon, with the tip placed $10 \mathrm{~cm}$ above the anus.

Electrophysiological recordings of the pelvic nerve afferent: The pelvic nerves innervating the lower gastrointestinal tract were identified with the aid of a binocular microscope. The pelvic nerve was sectioned, and its peripheral cut end was separated from surrounding connective tissues. The afferent activity from the whole pelvic nerve was recorded from its peripheral cut end. The nerve recording was performed within the abdominal cavity filled with paraffin oil using a platinum bipolar electrode connected to an amplifier (Biomedical Amplifier, MEG-1200, Nihon Kohden, Tokyo, Japan). The action potential of the pelvic nerve was displayed on an oscilloscope (SS-5215 Synchroscope, Iwatsu, Tokyo, Japan). All the signals were simultaneously recorded by a thermal array recorder (Thermal Array Recorder, Nihon Kohden) and stored on a digital tape recorder (PC208, SONY, Tokyo, Japan) or a personal computer equipped with an A-D converter (PowerLab 8/30, ADInstruments Pty. Ltd., Nagoya, Japan).

Drugs and DSS-induced enteritis: In the present study, it was first examined whether the pelvic afferent nerve was responsive to acidic and noxious stimulation applied to the colon. For this aim, acetic acid (volume, $0.5 \mathrm{ml}$ ), an inducer of acute enteritis $[13,14]$, with a concentration of $5 \%$ $(\mathrm{pH}=2.1), 10 \%(\mathrm{pH}=1.8)$ or $25 \%(\mathrm{pH}=1.4)$ was instilled into the colon. Furthermore, the difference in excitability of the afferent activity of the pelvic nerve was observed between normal and abnormal rats with or without DSS-induced enteritis. For rats in the DSS group with DSS-induced enteritis, DSS (molecular weight: $>500,000$ g, Sigma-Aldrich Pty Ltd., Castle Hill, Australia) solution with a concentration of $5 \%$ (wt/vol, diluted with tap water) was given as drinking water. In non-DSS group of rats, tap water without DSS was given ad libitum. Afferent activity from the pelvic nerve was recorded at 1 day and 8 days after DSS administration as drinking water in the DSS group and tap water in the nonDSS group. Body weight, diet and water consumption were measured daily. Hardness of stool and bleeding from the gastrointestinal tract were also observed daily.

A solution of capsaicin $(100 \mu \mathrm{g} / \mathrm{ml}, 0.5 \mathrm{ml})$, which is a selective stimulant to TRPV1 (transient receptor potential vanilloid type 1 receptor) on C-fiber endings and other cells, was administered into the colon to observe the change in nociceptive perception of the pelvic nerve in the DSS and non-DSS groups. Capsaicin (Sigma-Aldrich Pty Ltd.) was dissolved in vehicle (1\% Tween 80 and $10 \%$ methanol) and diluted by saline. Also, ruthenium red (RR) $(100 \mu \mathrm{g} / \mathrm{ml}, 0.5$ $\mathrm{m} l$ ), a potent transient receptor potential (TRP) channel antagonist, was administered intravenously at 2 min before capsaicin administration in some experiments in order to examine the effects on nerve excitation via transient receptor potential vanilloid 1 (TRPV1) by capsaicin in the DSS and
non-DSS groups.

The dosages of capsaicin and ruthenium red used in the present study were decided according to the past reports $[17$, 23, 28]

Data analysis: The discharge frequency recorded from the whole nerve was counted for every $30 \mathrm{sec}$ (impulses/30 $\mathrm{sec}$ ), and averaged for $90 \mathrm{sec}$ before instillation and for $600 \mathrm{sec}$ after colonic instillation of solutions. The values are expressed as means \pm SE. Whole nerve activity was measured by software (LabChart, ADInstruments Pty Ltd.) of a spike-height discriminator, setting the discriminating level to approximately 40 impulses/30 sec before solution instillation, and the change in discharge caused by the following experimental conditions was observed. Average discharge frequencies were counted and statistical analysis was performed by use of the Wilcoxon singed-rank test for comparison between before and after acetic acid instillation and capsaicin instillation in the non-DSS, DSS-1 and DSS-8 groups. Also, maximum responses to capsaicin were compared among the non-DSS, DSS-1 and DSS-8 groups by the Mann-Whitney $U$ test. Differences were considered statistically significant if the $P$ values were less than 0.05 .

All experiments were conducted in accordance with the Animal Experimentation Guidelines of the University of Tokyo and approved by the institutional Animal Care and Use Committee of the Graduate School of Agricultural and Life Sciences at the University of Tokyo.

\section{RESULTS}

Response to acetic acid: A representative record of the pelvic nerve afferent activity is shown in Fig. 1, which shows that the afferent discharge increased from immediately after instillation of acetic acid solution in each concentration (5, 10 and $25 \%$ ) group. The increase in mean frequency caused by acetic acid was shown to be dose-dependent (Figs. 1 and 2). In the 5 and $10 \%$ groups, the discharge frequency reached the maximum at $30 \mathrm{sec}$ after administration, and in $25 \%$ group maximum response was observed from 150 to $240 \mathrm{sec}$ after administration. In the $25 \%$ group, the increase in discharge frequency lasted for approximately $330 \mathrm{sec}$.

Response to capsaicin in DSS-induced enteritis: Responses of the pelvic afferents to capsaicin solution instilled into the colon are shown in Figs. 3 and 4. Discharge frequency was rapidly increased by the colonic instillation of capsaicin in all groups. The increase in discharge frequency was predominant for 0 to $90 \mathrm{sec}$ (early stage) immediately after the capsaicin instillation in all groups, whereas the discharge frequency increased again in the DSS-1 group approximately $300 \mathrm{sec}$ (late stage) after a short-term decrease at around 180 sec (Fig. 4). The increase in discharge frequency was most apparent in the DSS-1 group among the three groups at both early and late stages $(P<0.05)$, whereas no significant difference was present at early stage in DSS-1 group and at late stage in non-DSS group (Fig. 5). The discharge frequency was most high in DSS-1 group as compared with DSS-8 and non-DSS groups at late stage $(P=0.07$ between DSS- 1 and non-DSS groups). In the non-DSS group, the discharge 


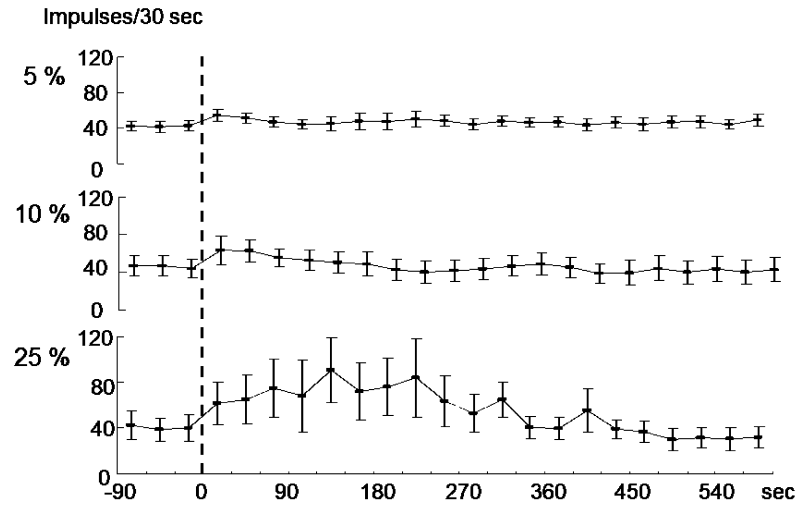

Fig. 1. Time-course changes of afferent activities in the pelvic nerve before and after the colonic instillation of acetic acid solution. All plotting data are expressed as means \pm SE of 6 rats. frequency returned the pretreatment level at approximately $300 \mathrm{sec}$ after the capsaicin instillation.

The maximum responses to colonic capsaicin instillation are shown in Fig 6. The discharge frequency during maximum response in all groups was significantly higher than that before instillation. The discharge frequency per $30 \mathrm{sec}$ during the maximum response was significantly higher in the DSS-1 group than in the other two groups.

Effects of RR on pelvic-nerve activity and on the response to capsaicin in DSS-induced enteritis: Time-course changes in the pelvic nerve afferent activity in response to capsaicin with or without RR pretreatment are shown in Fig. 7. In the non-DSS, DSS- 1 and DSS- 8 groups, there were no significant differences between the control and RR groups immediately before the capsaicin instillation, although the discharge frequency in the RR groups tended to be lower to a small extent than in the control groups without RR pretreatment. The colonic instillation of capsaicin significantly
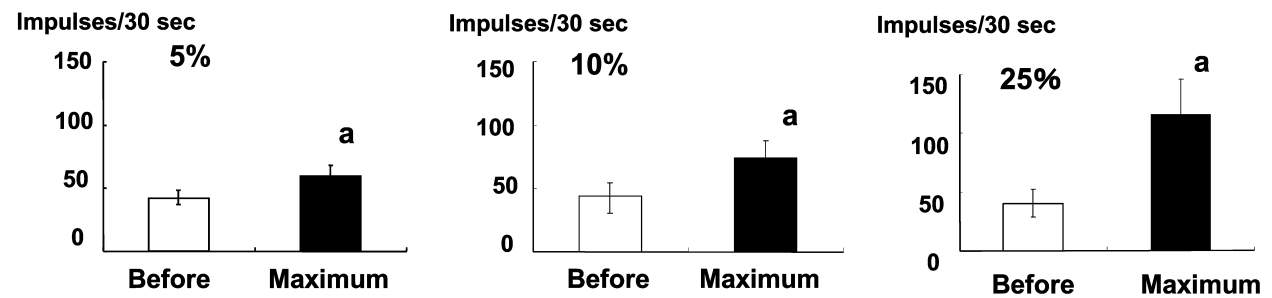

Fig. 2. Comparison of the maximum responses to 5 to $25 \%$ acetic acid solution. All data are expressed as means \pm SE of 6 rats. a: $P<0.05$ vs. before instillation of acetic acid.

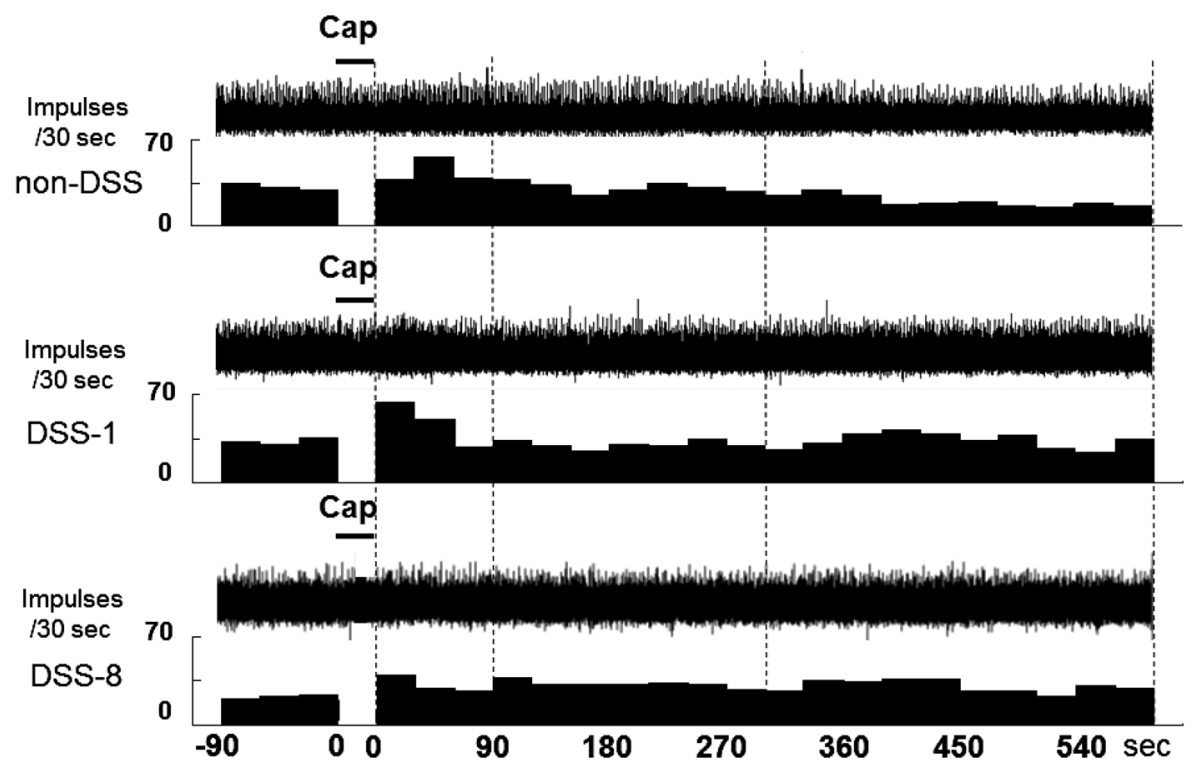

Fig. 3. Afferent activities of the pelvic nerve in response to the colonic instillation of capsaicin solution $(100 \mu \mathrm{g} / \mathrm{ml}, 0.5 \mathrm{~m} l)$ in rats. non-DSS: Recording from a normal rat in the non-DSS group. DSS-1: Recording from a rat in the DSS-group on one day after DSS administration. DSS-8: Recording from a rat in the DSS group on 8 day after DSS administration. The upper and lower graph in each panel for the groups shows the nerve recording and a histogram of the discharge frequency for every $30 \mathrm{sec}$, respectively. 


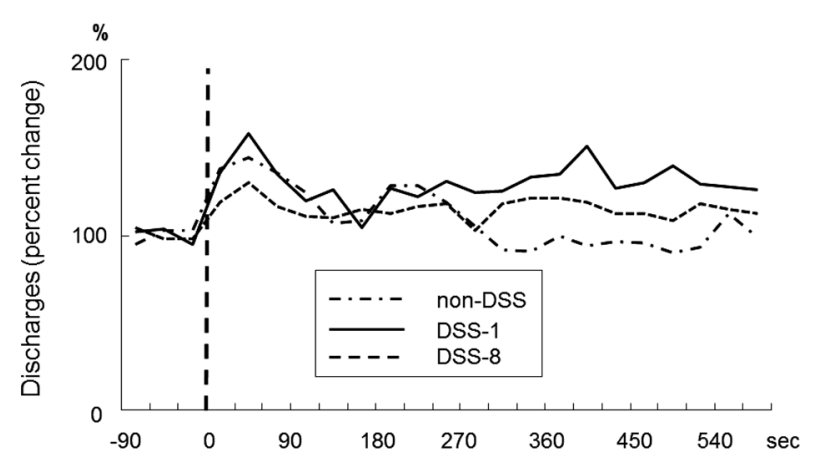

Fig. 4. Time-course changes of the pelvic nerve's afferent activity in response to the colonic instillation of capsaicin in the non-DSS, DSS-1 and DSS- 8 groups. The vertical dashed line shows the instillation of capsaicin $(100 \mu \mathrm{g} / \mathrm{m} l, 0.5 \mathrm{~m} l)$.

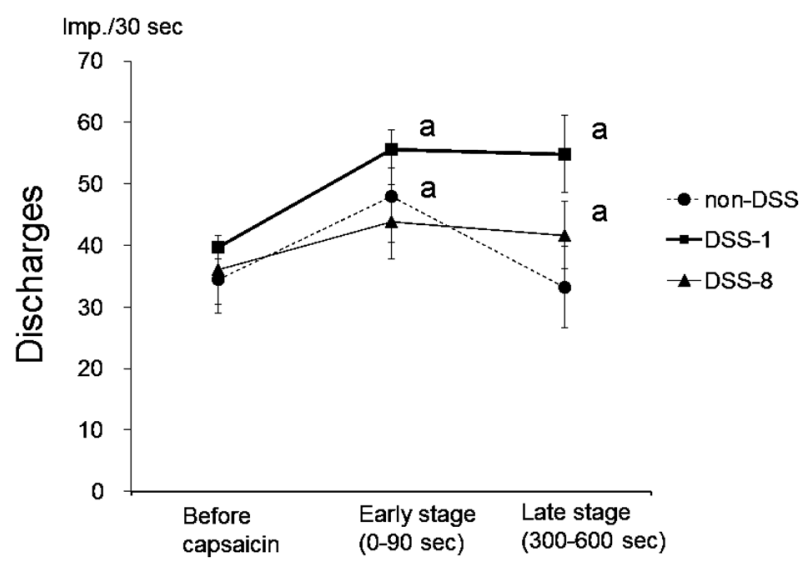

Fig. 5. Averaged changes in the pelvic nerve's afferent activity before and after the capsaicin instillation in the non-DSS, DSS-1 and DSS-8 groups. Each data was shown as an averaged value counted each $30 \mathrm{sec}$ for $90 \mathrm{sec}$ in "Before capsaicin" and "Early stage", and for 300-600 sec in "Late stage". a: $P<0.05$ vs. before capsaicin. All data are expressed as means \pm SE of 6 rats.

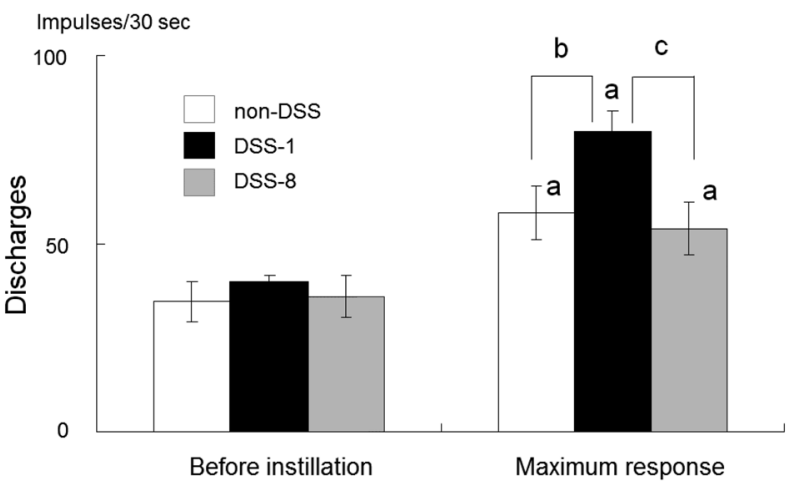

Fig. 6. Comparison of the maximum responses to colonic instillation of capsaicin. a: Significant difference $(P<0.05)$ compared with the before instillation. $\mathrm{b}$ : Significant difference $(P<0.05)$ between the non-DSS and DSS-1 groups. c: Significant difference $(P<0.05)$ between the DSS-1 and DSS-8 groups. All data are expressed as means \pm SE of 6 rats.

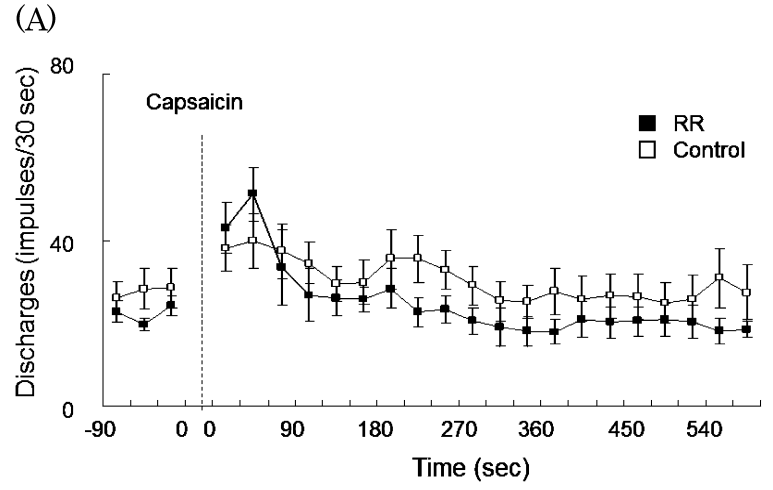

(B)
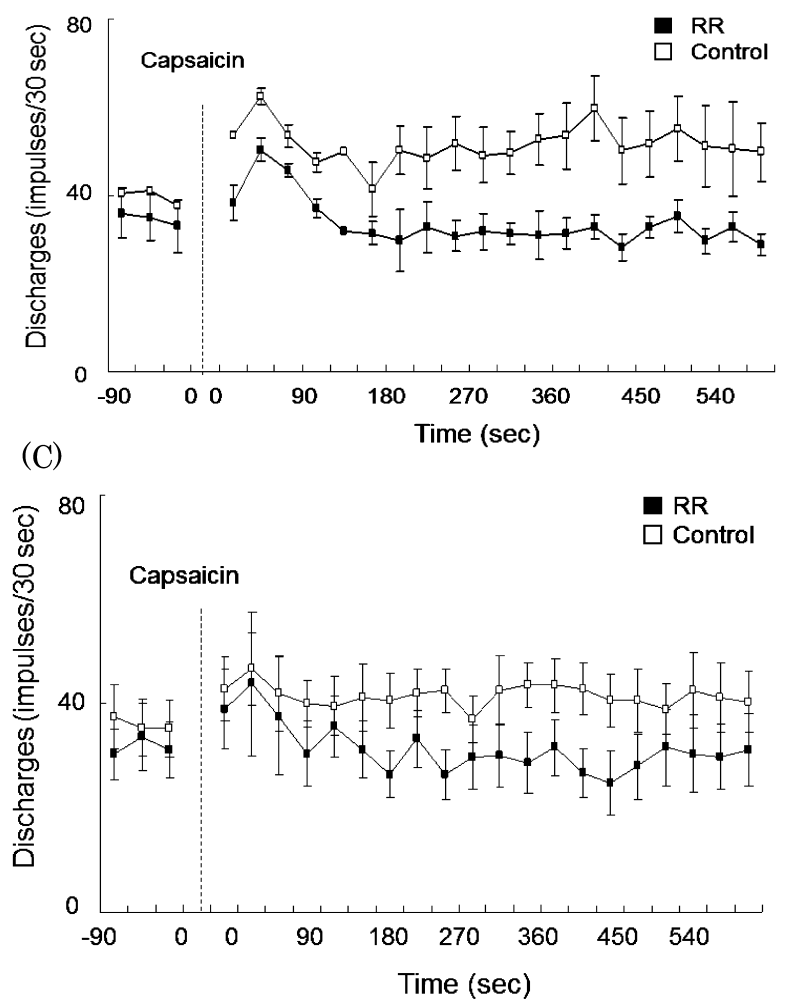

Fig. 7. Comparison of time-course changes in the pelvic nerve's afferent activity before and after colonic instillation of capsaicin with a pretreatment of ruthenium red in the non-DSS (A), DSS-1 (B), and DSS-8 (C). The vertical dashed lines indicate the instillation of capsaicin. Control: non-treatment of ruthenium red, RR: Ruthenium red pretreatment. All plotting data are expressed as means $\pm \mathrm{SE}$ of 6 rats.

increased the discharge frequency in all control groups (Fig. $7)$. The maximum responses to capsaicin were compared between the control and RR groups as shown in Fig. 8. The increase in discharge frequency by capsaicin instillation was significantly inhibited by pretreatment with RR in the DSS-1 and DSS- 8 groups (Fig. $8 \mathrm{~B}$ and $8 \mathrm{C}$ ), whereas a significant increase was observed in the control groups without RR pretreatment (Fig. 8A-C). Moreover, a significant decrease 

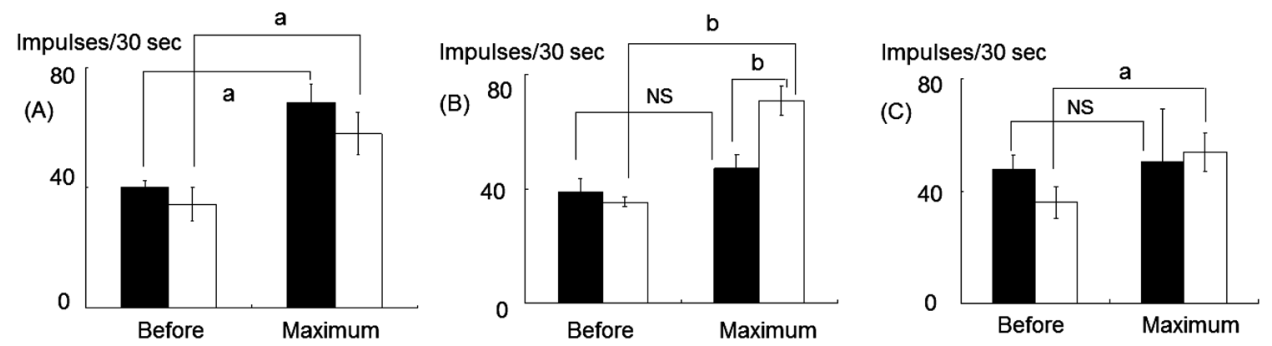

Fig. 8. Comparison of maximum responses to capsaicin between the RR and control groups in the non-DSS (A), DSS-1 (B) and DSS-8 (C) groups. $\llbracket$ : Ruthenium red group. $\square$ : Control group. Before: Before capsaicin instillation. Maximum: Maximum response after capsaicin instillation. a: $P<0.05$. b: $P<0.01$.NS: nonsignificant difference. All data are expressed as means \pm SE of 6 rats.
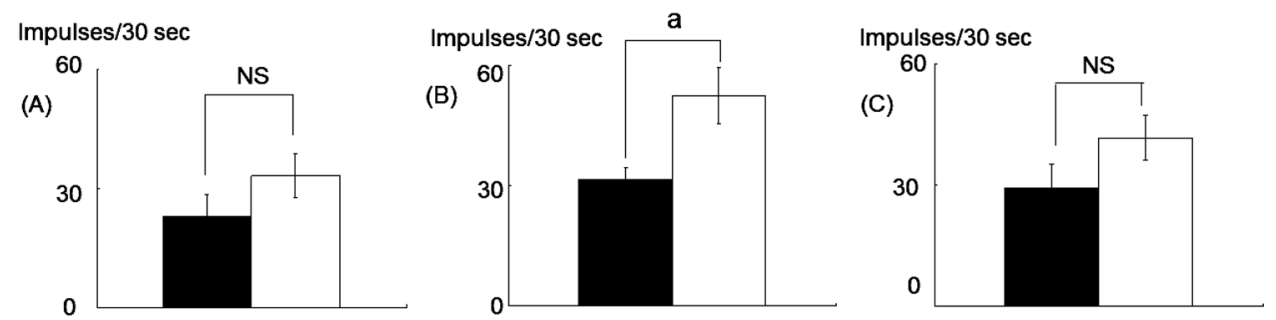

Fig. 9. Comparison of the pelvic nerve activity in the late stage at 300 to $600 \mathrm{sec}$ after colonic instillation of capsaicin in the non-DSS (A), DSS-1 (B) and DSS-8 (C) groups. a: $P<0.05$, $\mathbf{m}$ : Ruthenium red group. $\square$ : Control group. NS: nonsignificant difference. All data are expressed as means \pm SE of 6 rats.

in discharge frequency was observed in the DSS-1 group at the late stage, during 300 to $600 \mathrm{sec}$ after the capsaicin instillation, although no significant effect was present in the non-DSS and DSS-8 groups (Fig. 9).

\section{DISCUSSION}

The present study demonstrated that the afferents of the pelvic nerve were strongly stimulated by acetic acid and capsaicin instilled into the colon. This evidence suggested that this nerve has nociceptive properties that are highly responsive to acidic conditions and/or noxious chemical stimuli in the lower gastrointestinal tract. Although the effects of ruthenium red on the pelvic nerve activity was not examined in the present study, it has been known that protons activate TRPV1 in many DRG sensory neurons [6].

DSS has been widely used as a representative inducer of experimental colitis in experimental animals such as rats and mice, in which acute colitis characterized by histological damages to the intestinal epithelium, ulcer, hemafecia, diarrhea and increase in cytokines such as TNF $\alpha$ can be produced $[5,12,18,25]$ and the structural and ultrastructural changes have been shown to be similar to those in human colitis [7]. It has been reported that Sprague-Dawley rats, which were orally administered 4\% DSS solution, exhibited diarrhea at $6.14 \pm 0.69$ days (mean \pm SD) and hematochezia at $8.14 \pm 1.2$ days after the DSS was administered [7].

In the present study, the increase in discharge frequency in response to capsaicin in the DSS groups was shown to be larger than that in the non-DSS group. In particular, the responsiveness in the DSS-1 group to capsaicin was significantly greater than in the other two groups (Fig. 7). This evidence suggested that the nociceptive perception in the pelvic nerve to chemical irritants was already high one day after administration of DSS and that such augmented perception was decayed on the 8th day after DSS administration. This finding also indicated that the excitatory mechanism via TRP channels was enhanced at an early stage of colitis induced experimentally by DSS. It is considered that the cause of augmented nociception of the pelvic nerve to TRPV1 stimulants in DSS-induced colitis is associated with changes in inflammatory mediators that affect the activity of nociceptors. It has been reported that DSS produces increments in inflammatory mediators such as TNF $\alpha$, IL-8, MPO, COX-2, and NFKB in addition to oxidative and osmotic pressure stress in the colon $[5,9,11,12,19,20,22$, $26,27]$. A recent study regarding lung nociception showed that a pretreatment with $\mathrm{TNF} \alpha(50 \mathrm{ng} / \mathrm{m} l)$ for shorter than $24 \mathrm{hr}$ increased significantly the peak change in intracellular calcium concentration evoked by capsaicin in nodose and jugular ganglion neurons, whereas a pretreatment with the same concentration of TNF $\alpha$ for longer duration $(\sim 48 \mathrm{hr})$ did not further increase the response [10]. If TNF $\alpha$ had the same action on the pelvic nerve in the present study, the colonic increment of TNF $\alpha$ in the initial state of DSS-induced colitis may have enhanced the nociceptive response of the pelvic nerve to capsaicin. This may be supported by a report that a great increase, 70 to 80 -fold compared with control rats, in 
colonic TNF $\alpha$ mRNA expression was found on the first to second day after inducing colitis in rats using DSS [5]. This report by Dharmani et al. [5] also showed that the TNFa expression was reduced by 40 to 50 -fold between 3 and 8 days after inducing colitis using DSS.

The present study examined the pelvic afferent activity in response to capsaicin with or without pretreatment with RR which is a representative antagonist to TRP channels such as TRPV1. The increase in discharge frequency in response to capsaicin instillation was significantly inhibited by the pretreatment with RR in the DSS- 1 and DSS- 8 groups. This finding suggested that the responsiveness of the pelvic nerve to capsaicin was increased by the DSS-induced colitis and that this hypersensitivity was due to the increased exciting mechanism via TRP channels. The responsiveness to capsaicin in the DSS-1 group was maintained for longer, as shown in the late stages, while in the control and DSS-8 groups and also in rats with administration with acetic acid, such a prolongation of increased activity was not exhibited. The high activity in response to capsaicin in the late stage in the DSS-1 group was assumed to be derived from TRPV1mediating excitatory mechanisms in response to capsaicin because the late stage response was also abolished by the pretreatment with RR.

Such functional changes preceding severe morphological changes in colonic mucosa, which usually appear one week after DSS administration [7], might provide valuable information for understanding the initiation of chronic inflammation of the colon, in which such augmented nociception of C-fibers with TRP receptors in the initial stage of inflammation in the lower intestine might play an important role in promoting severe and chronic inflammation caused by peripheral and central neural mechanisms.

\section{REFERENCES}

1. Bielefeldt, K., Zhong, F., Koerber, H. R. and Davis, B. M. 2006. Phenotypic characterization of gastric sensory neurons in mice. Am. J. Physiol. Gastrointest. Liver Physiol. 291: G987-G997. [Medline] [CrossRef]

2. Branton, S. L., Reece, F. N. and Hagler, W. M. Jr. 1987. Influence of a wheat diet on mortality of broiler chickens associated with necrotic enteritis. Poult. Sci. 66: 1326-1330. [Medline]

3. Caterina, M. J., Schumacher, M. A., Tominaga, M., Rosen, T. A., Levine, J. D. and Julius, D. 1997. The capsaicin receptor: a heat-activated ion channel in the pain pathway. Nature 389: 816-824. [Medline] [CrossRef]

4. De Schepper, H. U., De Winter, B. Y., Van Nassauw, L., Timmermans, J. P., Herman, A. G., Pelckmans, P. A. and De Man, J. G. 2008. TRPV1 receptors on unmyelinated C-fibres mediate colitis-induced sensitization of pelvic afferent nerve fibres in rats. J. Physiol. 586 5247-5258. [Medline] [CrossRef]

5. Dharmani, P., Leung, P. and Chadee, K. 2011. Tumor necrosis factor- $\alpha$ and Muc2 mucin play major roles in disease onset and progression in dextran sodium sulphate-induced colitis. PLoS ONE 6: e25058. [Medline] [CrossRef]

6. Dubé, G. R., Elagoz, A. and Mangat, H. 2009. Acid sensing ion channels and acid nociception. Curr. Pharm. Des. 15: 1750-1766. [Medline] [CrossRef]
7. Gaudio, E., Taddei, G., Vetuschi, A., Sferra, R., Frieri, G., Ricciardi, G. and Caprilli, R. 1999. Dextran sulfate sodium (DSS) colitis in rats: clinical, structural, and ultrastructural aspects. Dig. Dis. Sci. 44: 1458-1475. [Medline] [CrossRef]

8. Hanauer, S. B. 2006. Inflammatory bowel disease: epidemiology, pathogenesis, and therapeutic opportunities. Inflamm. Bowel Dis. 12: S3-S9. [Medline] [CrossRef]

9. Herfarth, H., Brand, K., Rath, H. C., Rogler, G., Schölmerich, J. and Falk, W. 2000. Nuclear factor-kappa B activity and intestinal inflammation in dextran sulphate sodium (DSS)-induced colitis in mice is suppressed by gliotoxin. Clin. Exp. Immunol. 120: 59-65. [Medline] [CrossRef]

10. Hu, Y., Gu, Q., Lin, R. L., Kryscio, R. and Lee, L. Y. 2010. Calcium transient evoked by TRPV1 activators is enhanced by tumor necrosis factor- $\{$ alpha $\}$ in rat pulmonary sensory neurons. $A m$. J. Physiol. Lung Cell. Mol. Physiol. 299: L483-L492. [Medline] [CrossRef]

11. Iba, Y., Sugimoto, Y. and Kamei, C. 2002. Participation of mast cells in colitis inflammation induced by dextran sulfate sodium. Methods Find. Exp. Clin. Pharmacol. 24: 15-18. [Medline] [CrossRef]

12. Islam, M. S., Murata, T., Fujisawa, M., Nagasaka, R., Ushio, H., Bari, A. M., Hori, M. and Ozaki, H. 2008. Anti-inflammatory effects of phytosteryl ferulates in colitis induced by dextran sulphate sodium in mice. Br. J. Pharmacol. 154: 812-824. [Medline] [CrossRef]

13. Jurjus, A. R., Khoury, N. N. and Reimund, J. M. 2004. Animal models of inflammatory bowel disease. J. Pharmacol. Toxicol. Methods 50: 81-92. [Medline] [CrossRef]

14. Kim, H. S. and Berstad, A. 1992. Experimental colitis in animal models. Scand. J. Gastroenterol. 27: 529-537. [Medline] [CrossRef]

15. Kitajima, S., Takuma, S. and Morimoto, M. 1999. Tissue distribution of dextran sulfate sodium (DSS) in the acute phase of murine DSS-induced colitis. J. Vet. Med. Sci. 61: 67-70. [Medline] [CrossRef]

16. Kollarik, M. and Undem, B. J. 2004. Activation of bronchopulmonary vagal afferent nerves with bradykinin, acid and vanilloid receptor agonists in wild-type and TRPV1-/- mice. J. Physiol. 555: 115-123. [Medline] [CrossRef]

17. Kopczyńska, B. 2008. Role of VR1 and CB1 receptors in modelling of cardio-respiratory response to arvanil, an endocannabinoid and vanilloid hybrid, in rats. Life Sci. 83: 85-91. [Medline] [CrossRef]

18. Larrosa, M., Yañéz-Gascón, M. J., Selma, M. V., GonzálezSarrías, A., Toti, S., Cerón, J. J., Tomás-Barberán, F., Dolara, P. and Espín, J. C. 2009. Effect of a low dose of dietary resveratrol on colon microbiota, inflammation and tissue damage in a DSSinduced colitis rat model. J. Agric. Food Chem. 57: 2211-2220. [Medline] [CrossRef]

19. Liu, X. and Wang, J. M. 2011. Iridoid glycosides fraction of Folium syringae leaves modulates NF- $\mathrm{kB}$ signal pathway and intestinal epithelial cells apoptosis in experimental colitis. PLoS ONE 6: e24740. [Medline] [CrossRef]

20. Mayer, E. A., Naliboff, B. D. and Chang, L. 2001. Basic pathophysiological mechanisms in irritable syndrome. Dig. Dis. 19: 212-218. [Medline] [CrossRef]

21. McMahon, S. B. 2004. Sensitisation of gastrointestinal tract afferents. Gut 53: ii13-ii15. [Medline] [CrossRef]

22. Murano, M., Maemura, K., Hirata, I., Toshina, K., Nishikawa, T., Hamamoto, N., Sasaki, S., Saitoh, O. and Katsu, K. 2000. Therapeutic effect of intracolonically administered nuclear factor kappa B (p65) antisense oligonucleotide on mouse dextran 
sulphate sodium (DSS)-induced colitis. Clin. Exp. Immunol. 120: 51-58. [Medline] [CrossRef]

23. Naida, A. M., Ghosh, T. K. and Mathew, O. P. 1996. Airway protective reflexes elicited by laryngeal ammonia: Role of C-fiber afferents. Respir. Physiol. 103: 11-17. [Medline] [CrossRef]

24. Oliver, M. R., Tan, D. T., Kirk, D. R., Rioux, K. P. and Scott, R. B. 1997. Colonic and jejunal motor disturbances after colonic antigen challenge of sensitized rat. Gastroenterology 112: 1996-2005. [Medline] [CrossRef]

25. Ota, S., Yoshihara, S., Ishido, K., Tanaka, M., Takagaki, K. and Sasaki, M. 2008. Effects of proteoglycan on dextran sulfate sodium-induced experimental colitis in rats. Dig. Dis. Sci. 53: 3176-3183. [Medline] [CrossRef]

26. Rogler, G. and Andus, T. 1998. Cytokines in inflammatory bowel disease. World J. Surg. 22: 382-389. [Medline] [CrossRef]

27. Tsubouchi, R., Hayashi, S., Aoi, Y., Nishio, H., Terashima, S., Kato, S. and Takeuchi, K. 2006. Healing impairment effect of cyclooxygenase inhibitors on dextran sulfate sodium-induced colitis in rats. Digestion 74: 91-100. [Medline] [CrossRef]

28. Tsubone, H., Sant'Ambrogio, G., Anderson, J. W. and Orani, G. 1991. Laryngeal afferent activity and reflexes in the guinea pig. Respir. Physiol. 86: 215-231. [Medline] [CrossRef] 